\title{
DISCUSSION FOLLOWING PAPER BY KIPPENHAHN
}

Schwarzschild to Kippenhahn: May we ask Dr Kippenhahn for his personal estimate at this time as to whether 'plume' mixing will occur and be effective if in the evolution of a star an interface occurs between a carbon rich misture at the inside and a hydrogen rich mixture at the outside?

Kippenhahn: The position is not entirely clear. It will be necessary to take account of the mixing which occurs during the development of the instability.

What experience have you had of such interfaces?

Schwarzschild: In our calculations, mild hydrogen contact with carbon was reached in low mass stars.

Sugimoto: In the computation of the flickering helium shell burning by Prof. Schwarzschild and Härm, the effect of radiation pressure was not included. Since the entropy of radiation is rather large, the convection in the unstable helium shell might not reach the bottom of the hydrogen distribution. What do you think of this point?

Schwarzschild: Dr Sugimoto remembers correctly: our early model sequences for helium shell flash cycles were computed with neglect of radiation pressure. It is therefore not impossible that our results regarding the penetration of the convective tongue, caused by a helium shell flash, into the region containing hydrogen, may be affccted negatively by the proper inclusion of radiation pressure.

Sugimoto: I think that there is certainly a need of computations which take into account the entropy of radiation.

Schwarzschild to Kippenhahn: Did your early calculations of $5 M_{\odot}$ evolution include radiation pressure?

Kippenhahn: Yes. 